\title{
Stage III Breast Cancer AJCC v6
}

National Cancer Institute

\section{Source}

National Cancer Institute. Stage III Breast Cancer A/CC V6. NCI Thesaurus. Code C7769.

Stage III is divided into two stages IIIA and IIIB. Stage IIIA includes: (T0, N2, M0); (T1, N2, M0); (T2, N2, M0); (T3, N1, M0); (T3, N2, M0). Stage IIIB includes: (T4, any N, M0). T4: Tumor of any size with direct extension to (a) chest wall or (b) skin. N2: Metastases in ipsilateral axillary lymph nodes fixed or matted, or in clinically apparent ipsilateral internal mammary nodes in the absence of clinically evident axillary lymph node metastasis. N3: Metastasis in ipsilateral infraclavicular lymph nodes(s) with or without axillary lymph node involvement, or in clinically apparent ipsilateral internal mammary lymph node(s); and in the presence of clinically evident axillary lymph node metastasis or metastasis in ipsilateral supraclavicular lymph node(s) with or without axillary or internal mammary lymph node involvement. M0: No distant metastasis. (AJCC 6th Ed.) 